\title{
THE RADIOLOGY OF INTESTINAL OBSTRUCTION IN CHILDREN AND INFANTS
}

\author{
BY \\ J. H. MIDDLEMISS, M.D., D.M.R.D. \\ Assistant Radiologist, Royal Victoria Infirmary, Newcastle-on-Tyne
}

\section{Introduction}

Organic intestinal obstruction is often late in producing marked abdominal signs, and diagnosis tends to be delayed. Although nothing can replace a careful record of the history and thorough clinical examination, any further-diagnostic aid which is safe, simple, and effective is welcome in doubtful cases.

- Flat $x$-ray investigation of the abdomen in cases of suspected obstruction in adults and children has been used for some years, but not as extensively as it might be. Every clinician is anxious to avoid performing an unnecessary laparotomy, particularly in an ill child. Consequently there is a tendency to keep cases of suspected obstruction under clinical observation until a diagnosis can be made. This may mean that the child becomes acutely obstructed. If there is any investigation that will enable a diagnosis to be made at an earlier stage, that method should be adopted in all cases of doubt. Flat radiographs of the abdomen can give such information. There are, however, pitfalls in this form of investigation, and it is thought that the publication of some cases illustrating the scope and limitations of the method, may be of some value to paediatricians.

\section{Radiological Investigation in Intestinal Obstruction}

The diagnosis rests on the interpretation of abdominal gas shadows. Gas is normally present in the large bowel, and in infants commonly in the small bowel too. Over the age of three years gas is present in the small bowel, but unless some disorder of motility intervenes, and that disorder may be of a very minor nature, the gas is usually finely divided and intermixed with the fluid contents of the small bowel and rarely casts any shadow on a radiograph. Normally the contents of the small bowel are propelled towards the large bowel, and stasis does not occur. Thus, if gas is present in the small bowel, it will under normal conditions also be present in the large bowel. If gas is present in the small bowel and not in the large, if that part of the small bowel in which it is present is distended, and if in addition the existence of stasis in the small bowel can be demonstrated, then it can be deduced that some form of mechanical obstruction is preventing the onward passage of that gas. Obstruction of the large bowel can be diagnosed in a similar manner.

One of the most frequent causes of obstruction in infancy and early childhood is intussusception. Diagnosis is often difficult, especially in those cases where blood in the stools is a late symptom. Hellmer (1943) published a series of 110 cases of intussusception investigated and diagnosed by means of a barium enema. However, in a shocked child barium enema is not a procedure to be undertaken lightly; it will not improve the child's condition, and in any case is unlikely to demonstrate an ileoileal intussusception or a small-bowel obstruction from any other cause. Oral barium is to be avoided. The radiological procedure of taking straight films of the abdomen, however, is simple, in no way upsets the child, and, in a case of obstruction, whatever the cause may be, gives positive evidence in most cases.

Radiologically the various parts of the small and large bowel can be recognized with relative certainty. The upper part of the small bowel lies mainly in the upper abdomen and to the left side, and shows the mucosal folds or valvulae conniventes of the jejunum close together. The mid small bowel lies mainly in the umbilical region, and the mucosal folds are rather more widely spaced. The lower part of the small bowel lies mainly in the right flank and pelvis and shows the smooth walls of the ileum devoid of mucosal folds. The caecum and ascending colon lie in the right flank, the transverse colon crosses the upper abdomen, and the descending colon occupies the left flank; these parts of the colon show the characteristic haustrations or sacculations of the large bowel. The sigmoid colon occupies the pelvis and does not as a rule show the same saccular appearance. Occasionally, there may be some difficulty in differentiating the sigmoid colon from the ileum, or the jejunum from the transverse colon, but the difficulty can usually be overcome by taking a further radiograph with the patient in a different position.

The radiographic technique is to take two films of the abdomen of the child, one an antero-posterior 
projection with the child supine, the other a posteroanterior projection with the child either standing or suspended erect. The postero-anterior projection is used for the erect radiograph in order to produce less radiographic distortion of bowel shadows, and so to give a truer indication of the degree of distension.

As pointed out earlier, the physiological process is for the small bowel contents, both fluid and gaseous, to pass through the small bowel and on into the large bowel, a dynamic process. In the erect film, fluid levels indicate stasis at that site at the time that the exposure was made. The corresponding supine film will show in which parts of the bowel the gas is present, and if a mechanical block exists the most distal part of recognizable distended bowel will be in the region just proximal to the block. It should be remembered that in intussusception the obstruction is almost invariably at the proximal end of the process and so is in most cases a small-bowel obstruction.

Thus the radiological signs of mechanical obstruction in the small bowel are stasis in the small bowel, gaseous distension of the small bowel, and a complete absence of gas shadows from the large bowel. In some cases where obstruction is only partial, some gas bubbles may be present in collapsed bowel beyond the site of the obstruction. All gas shadows in the abdomen must be carefully studied; by identifying all the distended bowel, a relatively accurate localization of the site of the obstruction can be effected. By the same process a diagnosis of large-bowel obstruction can be established and localized. In a mixed series of over two hundred cases, adults and children, no case of large-bowel obstruction in a child was encountered other than those due to congenital anomalies such as an imperforate anus; in a previous paper the appearances of large-bowel obstructions in adults have been described (Middlemiss, 1948).

By this means obstruction can be demonstrated early, and elsewhere the author has published a case of intussusception in an infant diagnosed by straight radiography within twenty-four hours of the onset of symptoms (Middlemiss, 1948). The following cases have been selected to illustrate the points already mentioned.

Case 1. V.B., a girl aged 9 months, had a four-day history of vomiting and diarrhoea. During the last twenty-four hours the motions contained blood and mucus. On admission the baby was extremely ill and dehydrated. The abdomen was flabby and distended, but there was no palpable mass. Survey films (figs. 1 and 2) showed considerable distension of the small bowel (mostly the jejunum, but some ileum was recognizable), complete absence of gas shadows from the large bowel, and fluid levels present in the small bowel. This was regarded as evidence of a complete small-bowel obstruction in the mid or low small bowel. At operation an irreducible ileo-ileal intussusception was found; resection was performed, but the child died.

Case 2. K.A., a boy aged 5 months, had a cold and bronchitis fourteen days before admission. Thirty-six hours before admission he vomited and had obvious spasms of abdominal pain. Bowel action was normal at that time. The following day he started to vomit, passed blood in the motion, and was admitted to hospital. On examination he did not appear to be having much abdominal pain though he was obviously a poorly baby. There was no palpable tumour, and shortly after admission he passed a watery brown stool. He was thought to be suffering from gastro-enteritis. He took feeds for the next twelve hours without vomiting, and then passed another stool containing blood and started to vomit again. Survey films of the abdomen (figs. 3 and 4) showed very marked distention of a considerable length of small bowel, no gas in the large bowel, and, in the erect film, fluid levels in the small bowel. This was regarded as a complete intestinal obstruction occurring low in the small bowel. At operation an ileo-colic intussusception, the head of which had reached the hepatic flexure, was found and reduced. The patient made a good recovery.

Case 3. J.C., a boy aged 8 months, yomited intermittently for three days before admission. During this time he took some feeds but returned most of them. He had passed three stools a day, and for the last two days they had been hard but contained no blood. He had had no obvious attacks of abdominal pain. On admission he was an ill, dehydrated baby; the abdomen was a little distended, and it was considered that there was possibly a tumour in the right flank. It was not tender and nothing abnormal was detected per rectum. Survey films were taken, and the erect film (fig. 5) showed distension of the small bowel, mostly the ileum, containing fluid levels, and a complete absence of gas shadows from the large bowel; over the right ilium a single loop of ileum containing gas but collapsed and of normal lumen was demonstrated. It was considered that this was evidence of a partial low small-bowel obstruction. At operation the lower part of the ileum was found bound down to the posterior abdominal wall and obstructed by two enlarged lymph glands, and small tubercles were studded throughout the mesentery and generally throughout the abdomen. The obstruction was relieved, and the child recovered from the operation. When seen four months later the child was taking feeds normally.

Case 4. G.S., a boy aged 14 months, had been a healthy infant until three days before admission, when he was noticed to be pale and unhappy. He refused all food at that time and vomited, and subsequently continued to vomit frequently. He was completely constipated during the three days. 


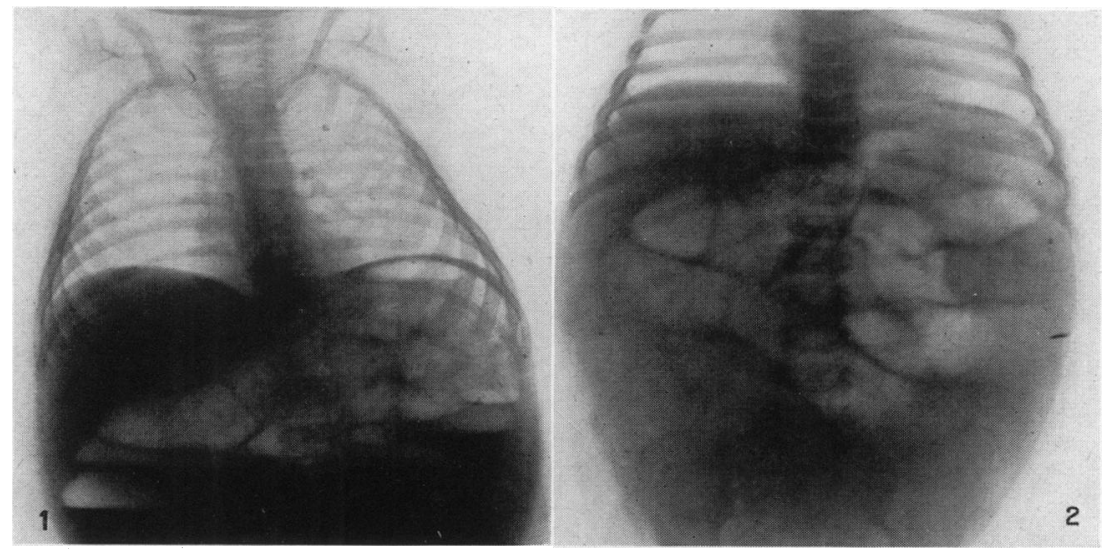

FIG. 1.-Case 1: erect film showing distension and fluid levels in small bowel.

Fig. 2. - Case 1: showing distended bowel to be mainly jejunum with some ileum and confirming complete absẹnce of gas from large bowel (supine film).

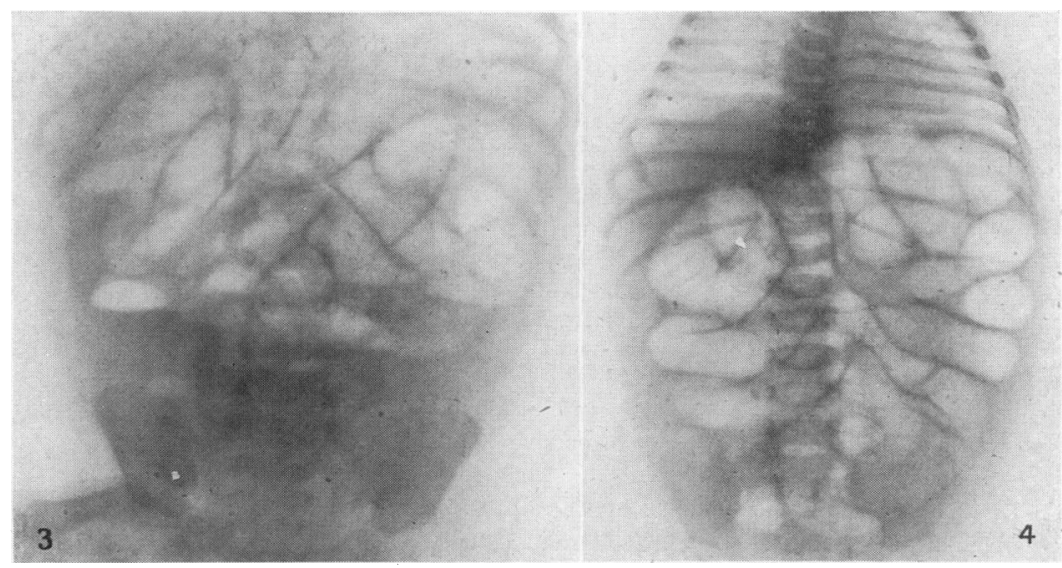

FIG. 3.-Case 2: erect film showing many distended loops of small bowel containing fluid levels.

FIG. 4.-Case 2: supine film showing that the distension involves a considerable length of small bowel, mainly ileum, and confirming complete absence of gas from large bowel.

\section{Plate I.}




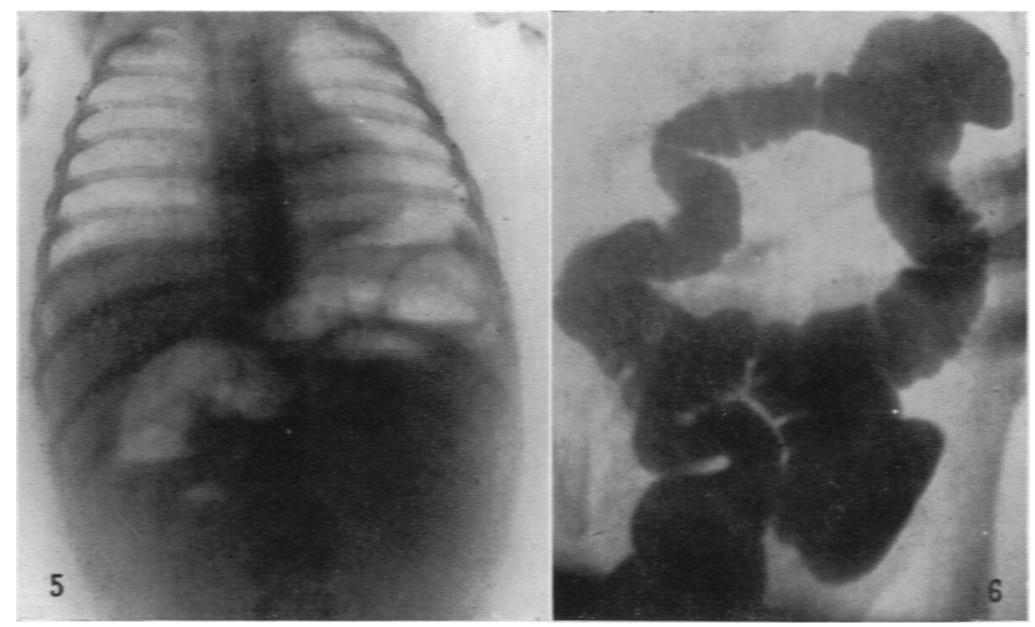

Fig. 5. - Case 3: erect film showing distension of loops of ileum containing fluid levels. There is no gas demonstrated in large bowel, but there is gas in a loop of ileum of normal lumen over the right ilium.

Fig. 6.-Case 4: taken during barium enema showing complete filling of large bowel.

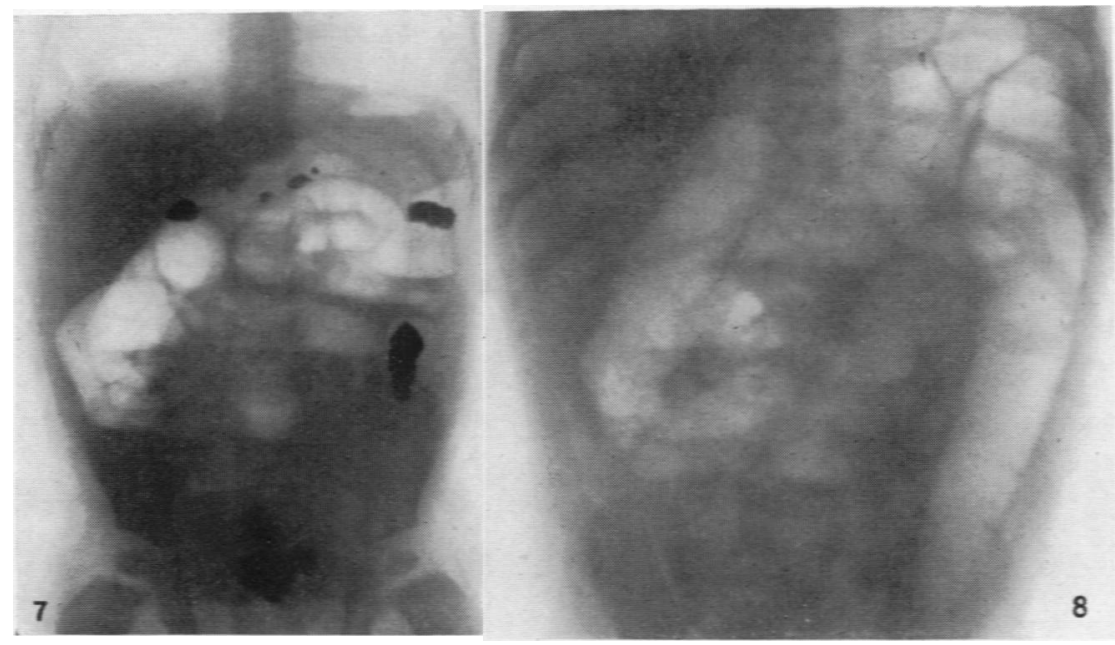

FrG. 7.-Case 4: erect film showing distension of loops of jejunum and ileum containing fluid levels, and complete absence of gas shadows from large bowel. Traces of barium remaining from the barium enema administered some hours earlier can be seen.

Fig. 8. - Case 5: erect film showing fluid levels in small bowel but in addition marked distension of large bowel.

Plate II. 


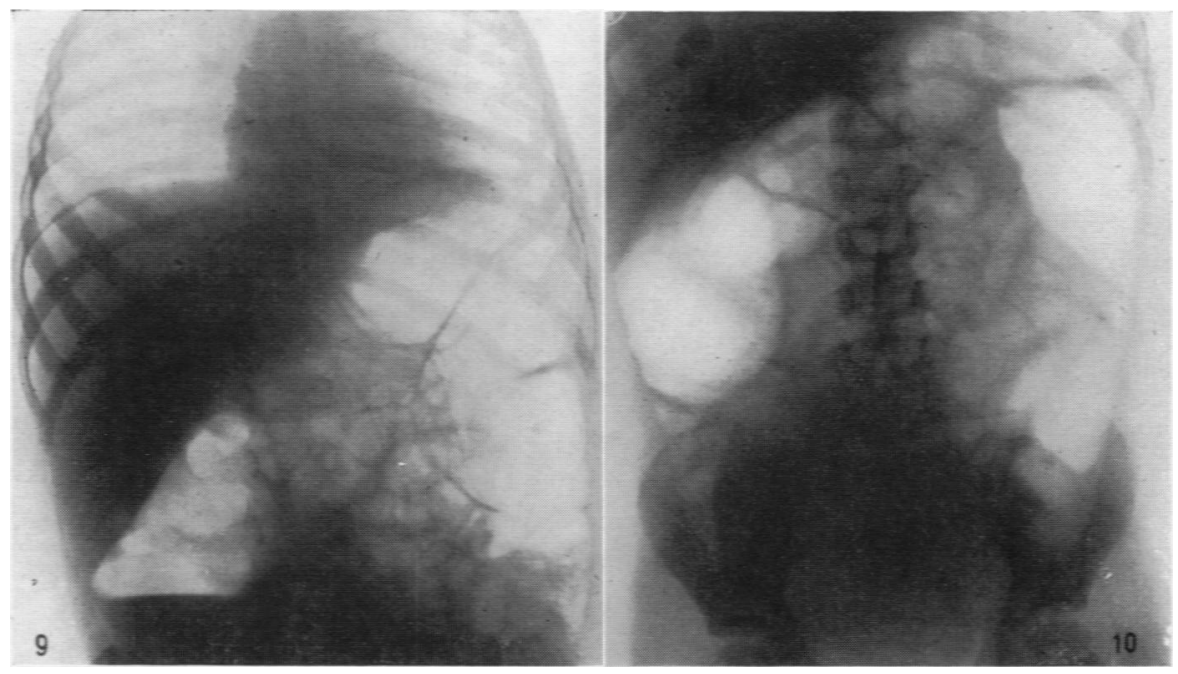

Fig. 9.-Case 6: erect film showing marked distension of many loops of bowel, some of them containing fluid levels. The length of bowel running along the left flank is shown not to have a fluid level at its lower end.

Fig. 10.-Case 6: supine film. Comparison with the erect film shows the fluid levels to be in small bowel and the ascending colon. Gaseous distension, however, extends as far as the distal end of the descending colon.

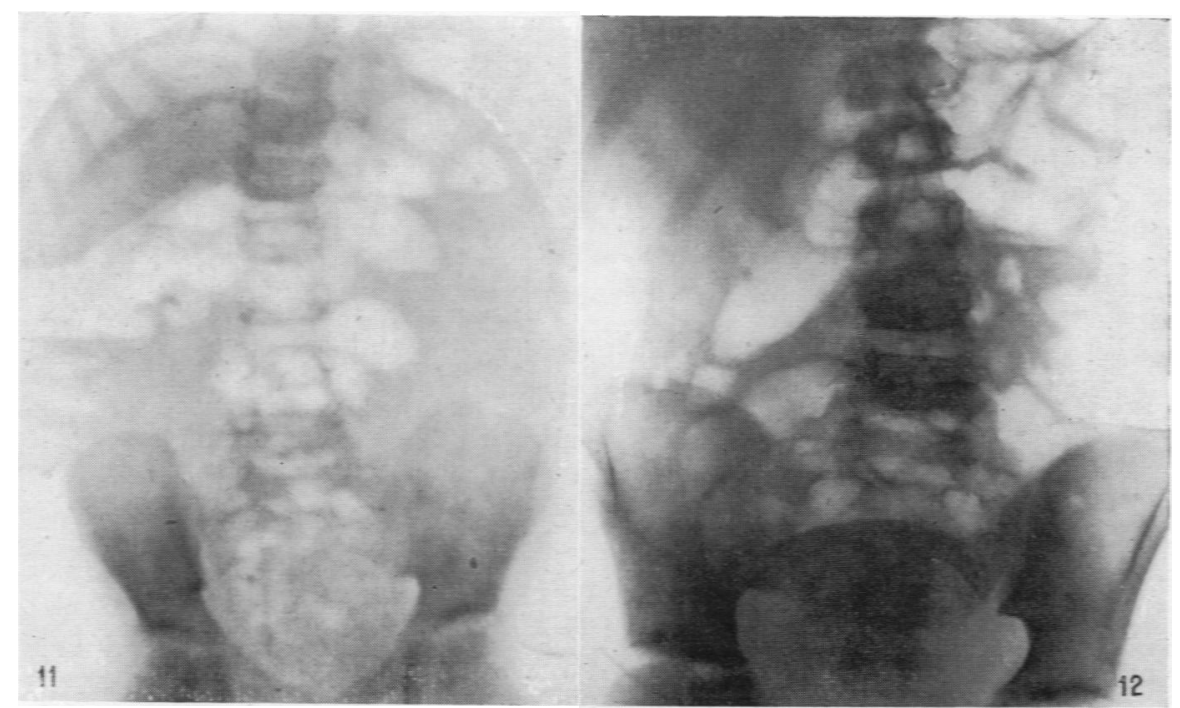

Fig. 11.-Case 7: erect film of a case of coeliac disease showing fluid levels in small and large bowel.

Fig. 12.-Case 8: supine film of an eight-year-old boy seven days after appendicectomy, showing distended loops of small and large bowel scattered throughout the abdomen.

Plate III. 
On admission to hospital he was a miserable, ill baby and appeared to be in pain. An enema removed a quantity of hard faeces from the rectum; there was no occult blood in the excreta. Intussusception being suspected, a barium enema investigation was carried out under screen control, but the large bowel was seen to fill completely (fig. 6). The infant returned to the ward, appeared to be improved, and took a feed; however, some hours later, as his condition again appeared to be deteriorating and vomiting had.recommenced, a survey film of the abdomen was taken (fig. 7). This showed distended loops of small bowel, jejunum, and ileum, with evidence of stasis of bowel contents and no evidence of any gas in the large bowel; thus there was conclusive evidence of a low small-bowel obstruction, probably complete. At operation a loop of ileum was found bound down to a Meckel's diverticulum by an adhesion, and the bowel immediately proximal to this was twisted about the adhesion. The obstruction was complete. The adhesion was severed, the obstruction relieved, and the child made a good recovery.

It is obvious that the survey would have been carried out more profitably as a preliminary investigation.

\section{Differential Diagnosis}

The demonstration of fluid levels in bowel is not an indication of intestinal obstruction; that is a point which cannot be too strongly emphasized. Fluid levels merely indicate that at the time of exposure the contents in that part of the bowel in which the fluid levels exist are static. It is essential to analyse all gas shadows, to determine the site and distribution of the fluid levels, to assess the degree of distension of the bowel, and to establish the fact that gas does not exist in any quantity in the bowel beyond that part in which the fluid levels are present. In other words the state of affairs must be seen as a whole, and one isolated sign must not be fastened on and misinterpreted.

Case 5. Fig. 8 is the erect film of a boy of 6 months who presented with a history of three days' diarrhoea with green offensive motions, and obvious spasms of abdominal pain; ten hours before admission he passed dark red blood per rectum and began to vomit. After admission no stool was passed but vomiting continued. The baby was pale and dehydrated. No abdominal tumour was palpable, but he was considered possibly to have intussusception. The film showed fluid levels in the small bowel with some distension of the small bowel; but, in addition there was very considerable gaseous distension of the large bowel, in which no fluid levels could be detected. With such distension of the large bowel there obviously could not be a small-bowel obstruction. In fact after administration of sulphamezathine the child recovered rapidly from what had been a severe gastro-intestinal upset of an indeterminate nature. It should be noted that in the earlier stages the radiological investigation, though presenting certain features which might have been misinterpreted to indicate an obstructive lesion, in fact provided evidence to the contrary.

Case 6. Figs. 9 and 10 are the erect and supine films respectively, of a boy aged 4 years. He was admitted to hospital as a case of intestinal obstruction, acute abdominal pain. having started about midday the same day. He was a flushed child, crying continuously, retching occasionally, but not actually vomiting. There was generalized abdominal distension with resistance on palpation but no obvious rigidity or tenderness. There were marked borborygmi. The x-ray investigation was carried out at 3.0 a.m., and the clinicians did not call in the radiologist on duty. On their own interpretation of the films, backed by the clinical signs and symptoms, they decided that the child was obstructed and they carried out an exploratory laparotomy. No obstruction was found. The films show distension of the small bowel and of the large bowel as far as the sigmoid colon; there are fluid levels in the small bowel and in the right half of the large bowel. This shows quite clearly that there is some intestinal disorder, but it equally clearly rules out the possibility of obstruction. The child subsequently developed left-sided pneumonia with effusion, and made a normal recovery.

This case shows the value of $x$-ray investigation in such disorders, and also the necessity for having a radiological opinion.

Case 7. Fig. 11 is the erect film of a child aged 4 years who had coeliac disease. A routine barium follow-through investigation was carried out, and scout films of the abdomen were taken beforehand. Clinically there is little likelihood of a case of coeliac disease being investigated for potential intestinal obstruction. It is of interest, however, to demonstrate the existence of fluid levels in such a case: the author has consistently shown this in such cases. Analysis of the gas shadows in the supine film of this case showed that the distribution was such that it could not under any circumstances be due to a mechanical obstruction.

These three cases serve to show that fluid levels may occur in bowel under many other conditions than those produced by mechanical obstruction. There is, however, one important class of cases in which they may occur and which it is important to differentiate from mechanical obstructions, that is, postoperative distensions. A postoperative distension may be traumatic, that is, a result of the handling of bowel during a laparotomy, or infective, for example, due to peritonitis. In most cases the process is a temporary or transitory one lasting from a few hours to three or four days. It may resolve, or it may become generalized, the so-called 
'paralytic ileus.' Whatever the stage of the condition, it is primarily the inhibitory result of active stimulation of the sympathetic nerve supply to bowel-wall muscle. The radiological appearances show either a segmental or a general distribution; if segmental, the small and large bowel in one particular region in the abdomen are involved; if general, then the small and large bowel throughout the whole abdomen are involved. This involvement shows a distension of bowel by gas; the distension may vary from the size of the normal lumen of bowel to a very marked dilatation. In an erect film of the condition there may be fluid levels, and if so they are scattered throughout both the small and large bowel, showing no tendency to be restricted to the proximal end of the alimentary canal, as they are in mechanical obstruction.

Case 8. Fig. 12 is the supine film of such a case of inhibition ileus of infective origin. It is of a boy aged 8 years on whom seven days previously appendicectomy with drainage had been performed; peritonitis had ensued, with considerable abdominal distension. Subsequently a pelvic abscess was drained. The film shows generalized distension of the small and large bowel with some ' layering' between the loops of bowel, probably due to the peritoneal effusion. The erect film (unsuitable for reproduction) showed fluid levels with a similar distribution. The distribution of the gas shadows excludes the possibility of a mechanical obstruction.

It is of the utmost importance in such cases of postoperative distension to be able to differentiate between an inhibition ileus and a mechanical obstruction. In the event of a mechanical obstruction (due, for example, to adhesions) developing as a postoperative complication, its early diagnosis and differentiation from a simple postoperative distension may save life. This radiological investigation, while not necessarily infallible, is often a means of demonstrating earlier than by clinical observation the fact that a mechanical obstruction is developing or has developed. It is recommended that survey films of the abdomen be taken in all cases of abdominal distension persisting or developing after the fifth postoperative day. In doubtful cases this investigation should be repeated at intervals of twenty-four hours.

\section{Summary}

1. The appearances of mechanical obstruction of the bowel are: (a) stasis, as shown by the presence of fluid levels; (b) distension of bowel by gas; and (c) absence of gas shadows, or the demonstration of collapsed bowel distal to the obstruction. The site of the obstruction can be localized just distal to the most distal part of distended bowel.

2. Fluid levels may occur in bowel due to other conditions. Of these the most important is inhibition ileus occurring as a postoperative complication. In this, the small and large bowel are involved, either in one segment of the abdomen or throughout the whole abdomen, and the distension and fluid levels are not restricted to the proximal side of a block as in mechanical obstruction, but are distributed through the whole alimentary canal.

3. In a paediatric department, or indeed in any hospital, the contributions which this technical procedure has to make as an aid to clinical diagnosis may be listed as follows:

(a) it is essentially a time-saving factor, often enabling the establishment of earlier diagnosis and so providing for earlier surgical treatment before acute obstruction develops;

(b) it may provide a diagnosis in many cases in which the history and clinical signs and observation have failed to do so; this applies particularly to infants, and in many instances it may be the means of establishing a diagnosis when the alternative may be the spending of a further valuable twenty-four hours in clinical observation, which in turn may only lead to a delayed exploratory laparotomy;

(c) it is especially helpful in the observation of postoperative distension, particularly after the fifth day, when the clinician is on the look-out for the possible development of mechanical obstruction as a postoperative complication.

\section{REFERENCES}

Ascroft, P. B., and Samuel, E. (1941). Brit. J. Radiol., $14,11$.

Bigss, A. D., and Pontius, G. V. (1947). J. Pediat., $30,306$.

Hellmer, H. (1943). Acta radiol. Stackh., 24, 235.

Middkmiss, J. H. (1948). Brit. J. Radiol. (In the press.) 